\title{
Kernos
}

Revue internationale et pluridisciplinaire de religion grecque antique

9| 1996

Varia

\section{Le culte de Dionysos à Argos}

\section{Marcel Piérart}

\section{(2) OpenEdition \\ Journals}

\section{Édition électronique}

URL : http://journals.openedition.org/kernos/1189

DOI : 10.4000/kernos. 1189

ISSN : 2034-7871

\section{Éditeur}

Centre international d'étude de la religion grecque antique

\section{Édition imprimée}

Date de publication : 1 janvier 1996

ISSN : 0776-3824

\section{Référence électronique}

Marcel Piérart, «Le culte de Dionysos à Argos », Kernos [En ligne], 9 | 1996, mis en ligne le 21 avril 2011, consulté le 24 septembre 2020. URL : http://journals.openedition.org/kernos/1189 ; DOI : https://doi.org/10.4000/kernos.1189 


\title{
Revue des Livres
}

\author{
1. Compte rendu critique \\ Le culte de Dionysos à Argos
}

\author{
par Marcel Piérart
}

Giovanni CASADIO, Dioniso e Semele: morte di un dio e resurrezione di una donna, in F. BerTi (éd.), Dionysos. Mito e mistero, Commacchio, 1991, p. 361-377.

-, Les rites d'adolescence et les mystères, in L'initiation, Montpellier, 1992, I, p. 209-213.

-, Storia del culto di Dioniso in Argolide, Rome, Gruppo editoriale internazionale, 1994. 1 vol. $14,5 \times 22,5 \mathrm{~cm}, \mathrm{XI}+371$ p., 8 ill. (Filologia e critica, 71). ISBN 888011-026-8.

Le culte de Dionysos en Argolide a donné lieu à des rites et des mythes anciens et originaux. Les travaux que G. Casadio lui a consacrés récemment sont issus d'une thèse de doctorat soutenue en 1981. L'érudition qui y est déployée, et qui transparaissait déjà dans des travaux plus anciens, désigne leur auteur comme l'un des spécialistes du dionysisme et de ses rapports avec les cultes à mystère et les doctrines qui s'y rapportent. Le livre, que complètent deux articles parus presque en même temps, est consacré, selon les mots de l'A. au mare magnum de l'Argolide (p. III). La notice biographique reproduite sur la couverture résume l'itinéraire spirituel suivi : «Muove della comparazione e dalla tipologia storica per giungere a una interpretazionecomprensione che salvaguardi le esigenze della critica storico-filologica. » Les remarques suivantes entendent se cantonner au strict point de vue de la critique historique et philologique. Elles se concentrent sur les cultes et les légendes de l'État-cité d'Argos. C'est ainsi qu'on laissera généralement de côté la question, chère à l'A., des rapports entre les mythes et les rites étudiés avec l'orphisme et certaines interprétations iconographiques (par ex. l'ivoire mycénien du Musée national, p. 37-42, l'amphore 1760 de Naples, p. 100-102, 107 , le cratère de bronze de Dhervéni, p. 210-215 et le cratère à volutes de Ceglie, p. 265-284) portant sur des documents dont les liens avec le culte de Dionysos en Argolide ne sont pas évidents : il n'est pas possible, en effet, de rendre un compte exact de tout ce que contient ce très riche ouvrage.

Le livre comprend une introduction et trois chapitres.

I. Dans l'introduction (p. 1-50), après de brèves considérations sur l'histoire et la géographie, qui visent à affirmer la continuité des cultes et des traditions en Argolide, l'A. s'étend sur le culte de Dionysos pendant l'âge du bronze. Tout 
en soulignant le caractère hypothétique de reconstructions qui ne reposent que sur quelques mentions à Pylos et à Kydonia, C. semble admettre l'existence d'une triade pylienne « dans laquelle deux divinités adultes représentant d'une façon ou d'une autre les deux sexes sur un plan d'égalité absolue apparaissent en relation avec une figure divine masculine (peut-être celle qui est appelée ailleurs Dionysos) qui leur est subordonnée dans un rapport de filiation... » (p. 23). Des poèmes d'Alcée et de Sapho conserveraient la trace d'une triade semblable à Lesbos, qui pourrait remonter à l'âge du bronze.

Le manuscrit du livre qui en est issu a été achevé en mars 1992. Plusieurs études consacrées au problème de la continuité entre la culture mycénienne et la culture grecque, d'une part, à la géographie de l'Argolide de l'autre ne lui ont pas été accessibles : D. MUSTI (éd.), La transizione dal Miceneo all' Alto Arcatsmo (Rome 1991); M. PIÉRART (éd.), Polydipsion Argos (Paris-Fribourg 1992); E. ZANGGER, The Geoarchaeology of the Argolid (Berlin 1993). Ce livre est le produit du remaniement d'une thèse publiée sous le nom de E. Finke en 1988 (Landscape Evolution of the Argive Plain). Pour la géographie, au lieu de Tomlinson (1972), peu satisfaisant, on aurait déjà pu se référer à R. BALADIÉ, Le Péloponnèse de Strabon (Paris 1980), p. 87-88.

La question de la continuité des cultes est une bouteille à l'encre. Sur ce point, je ne suivrai pas Casadio. Parler des invasions doriennes comme un fait historique, ou invoquer les mythes de fondation de Lesbos pour justifier d'une continuité entre l'Argos mycénienne et la Lesbos éolienne, relève d'une lecture historiciste des légendes qui n'est plus de mise aujourd'hui. Dionysos apparaissant dans les tablettes de Pylos et de La Canée, rien n'empêche a priori son culte de remonter très haut en Argolide, mais dans l'état actuel de notre documentation, nous ne remontons pas au-delà de l'époque archaïque, même si les rites et les mythes très archaïques de Lerne permettent de supposer une très haute antiquité ( $c f$. M. PIÉrarT, in D. Musti, op. cit., p. 134-135). En tout état de cause, l'image - vraie ou fausse - qu'on se fait des rapports de Dionysos avec Zeus et Héra à l'époque mycénienne n'a pas de répondant à Argos au premier millénaire: Dionysos n'y apparaît jamais que comme un Dieu adulte, luttant contre Persée à la tête des Haliai, ou ramenant Sémélé des enfers.

II. Le premier chapitre (Proitides et Agriania, p. 51-122) est consacré à la célèbre légende de la folie des filles de Proitos et de leur cure par le devin Melampous. La difficulté vient de ce que trois divinités (Héra, Dionysos, Artémis) interviennent dans la légende des Proitides. Alors que la tradition dionysiaque est déjà connue d'Hésiode, Acousilaos a innové (innovò p. 56) en introduisant Héra à la place de Dionysos. La légende tout entière tourne autour de la figure de Melampous. Figure sui generis, il est un propagandiste par excellence du culte de Dionysos, mais dans la légende des Proitides, il garde une certaine autonomie par rapport au dieu. Il n'est pas sans rapports avec Apollon, avec qui Dionysos a d'ailleurs plus d'un lien. Le rite des Agriania, lié au mythe des Proitides, renvoie aux Agrionia de Béotie, où le rituel, associé à Dionysos est mieux connu. L'A. semble admettre que « les mythes et les rites liés aux Proitides s'insèrent aisément dans la thématique des initiations fémi- 
nines qui, dans ce cas au moins, se présentent naturellement comme un prius par rapport à l'orgiasme bacchique (p. 111). »

Sur Melampous et les filles de Proitos, on consultera aussi désormais M. JOST, La légende de Mélampous, in M. PIÉRART (éd.), Polyditpsion Argos (1992), p. 173-184; K. DOWDEN, Death and the Matden (Londres-New-York 1989), p. 71-115. P. BONNECHERE, Le sacrifice bumain en Grèce ancienne (Liège 1994), p. 192-201.

Il est difficile, quand deux versions d'un mythe sont étroitement apparentées, de dire laquelle est antérieure à l'autre. Selon [Apollodore] (II, 2, 2), Hésiode aurait donné la version impliquant Dionysos, Acousilaos, celle qui est

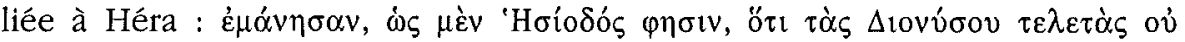

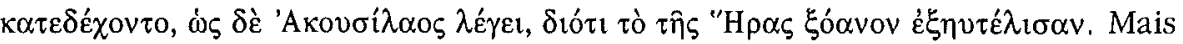
selon Probus, in Verg. Ecl. VI 48 ( $c f$. fr. 131 M.-W.), Hesiodus docet [...] quod Iunonis contempserunt numen. Les fragments du Catalogue ne permettent pas de trancher absolument, mais semblent plutôt impliquer l'intervention d'une déesse, vraisemblablement Héra : W. BURKERT, Homo Necans (tr. angl. 1983), p. 169 n. 6. On n'admettra pas facilement l'idée qu'Acousilaos a innové : il rapporte plutôt une tradition locale. Celle-ci pouvait être très ancienne. La figure complexe de Melampous est commune aux diverses variantes du mythe. Il n'a pas de rapport nécessaire avec Dionysos : il passait aussi pour avoir fondé des cultes d'Apollon et d'Artémis. Je croirais volontiers que la version impliquant Héra est la plus ancienne. Ceci s'accorderait bien avec la définition des Agr(i)ania argiennes qu'Hésychius présente comme une vexvoí $\alpha$. L'expression

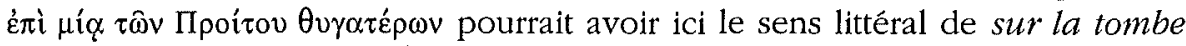
d'une des filles de Proitos. À Sicyone, en tous les cas, la version qui circulait au IVe siècle et y expliquait la présence sur l'agora de la tombe d'Iphinoé, impliquait Héra et Melampous ( $S E G, X V, 195)$. Les analogies avec des récits et des rites analogues impliquant ailleurs Dionysos seraient à l'origine de la seconde variante, peut-être dès la Mélampodie pseudo-hésiodique - mais c'est loin d'être sûr -, une variante qu'ont dû favoriser par la suite les difficultés chronologiques qu'impliquait la mise en rapport de Melampous avec les filles de Proitos.

Selon Dowden, les traditions argiennes, sicyoniennes, etc. n'ont que des racines superficielles. Il est vrai qu'en dehors du xoanon d'Héra (dont on ne sousestimera pas l'importance dans les traditions argiennes), la seule curiosité qu'on peut leur associer sur le territoire d'Argos sont les thalamoi près de Tirynthe (Paus., II, 25, 9). Il pourrait d'ailleurs s'agir là d'une identification érudite relativement tardive. Mais l'utilisation de la légende comme mythe explicatif des Agr(i)ania précède sans aucun doute l'époque d'Acousilaos. Le succès de la légende des filles de Proitos dans le Péloponnèse peut s'expliquer par un phénomène de diffusion "horizontale » à l'époque archaíque (rivalité Sicyone-Argos, par exemple). Il se pourrait cependant qu'elle ait déjà été très répandue avant le processus d'individuation qui accompagna la formation des États-cités. Quoi qu'il en soit, la version impliquant Héra était parfaitement à sa place à Argos. 
III. Le chapitre deux (p, 123-222) est consacré à Dionysos et Ariane. Partant du passage de Pausanias cité ci-dessous, Casadio montre la diversité des traditions relatives à Dionysos et Ariane. Elle paraît être une ancienne déesse (qu'il faut peut-être reconnaître dans la potnia du Labyrinthe des tablettes de Cnossos), qui après une hiérogamie avec Dionysos, serait morte de mort violente. L'enquête approfondie sur les traditions et les cultes en Crète, dans les îles (Céos, Délos, Naxos), à Chypre, à Lemnos, Oinoé de Locride et à Athènes conduit à penser que « dans l'héroïne que se disputent Thésée et Dionysos, on peut reconnaître la forme mythologiquement évoluée d'une divinité féminine de la nature ou de la végétation, [...] notamment des arbres (p. 205)». Les moments forts des vicissitudes de l'héroïne sont ses noces avec Dionysos et sa mort. L'hiérogamie existe surtout dans les mythes et, peut-être, dans certains rituels. Le problème de la mort d'Ariane sous diverses formes (y compris la pendaison, qui a conduit à voir en elle une divinité des arbres) est plus épineux. La question de la signification de l'hiérogamie dans le culte de Dionysos, après l'apparition des cultes à mystères, demeure également douteuse.

Sur les traditions, voir maintenant la typologie de C. CALAME, Thésée et l'imaginaire athénien (Paris 1990), p. 106-116. Ariane et Aphrodite (Ourania) : V. PIRENNE-DELFORGE, L'Apbrodite grecque (Liège 1994), p. 165-167 (Argos), 349 (Amathonte), 375, 396, 420, 456 (Délos).

Si on se place dans la perspective de l'étude du panthéon d'Argos, il importe de séparer soigneusement la géographie mytbologique, qui explique les particularités locales, de l'histoire réelle d'un culte et de ses origines. L'épithète argienne de Dionysos Kṕ́бı s renvoyait indiscutablement à la Crète pour les anciens et invite donc à insérer les traditions argiennes dans un ensemble de traditions connues autrement. Cela suffit-il à affirmer que « le culte de Dionysos crétois à Argos, près de la tombe d'Ariane, prouve certainement l'existence d'une tradition de l'arrivée de Dionysos de la mer et de Crète, en compagnie d'Ariane et des autres femmes, comme cela est implicite dans le récit même de Pausanias (p. 164) »? Les paragraphes II, 23, 7-8 de Pausanias décrivent des curiosités mentionnées hors topographie et placées, après les promenades urbaines, au moment où il s'apprête à monter à l'Acropole. Il peut être utile de revenir au texte du Périégète :

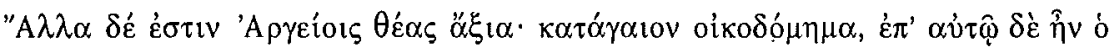

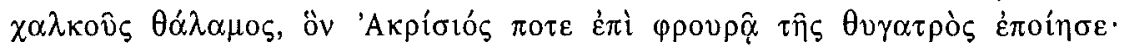

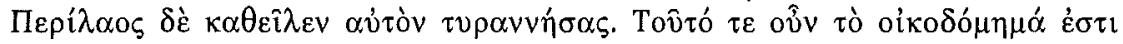

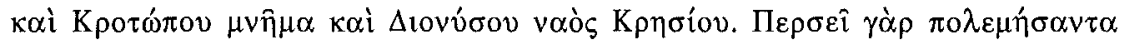

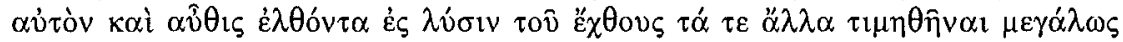

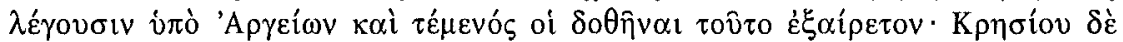

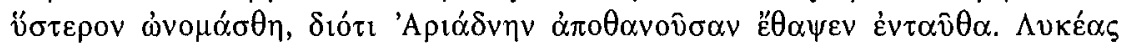

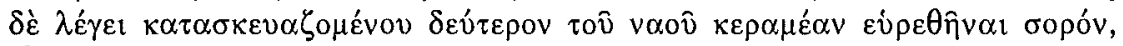

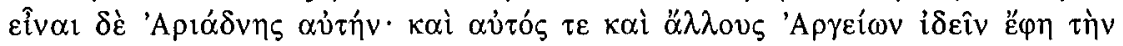

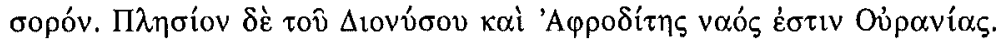


Les Argiens possèdent d'autres curiosités qui méritent d'être vues: une construction souterraine était surmontée par la chambre de bronze bâtie autrefois par Acrisios pour y enfermer sa fille. Perilaos l'a fait abattre lors de sa tyrannie. Cette construction est à la fois la tombe de Krotôpos et le temple de Dionysos Krésios. En effet, après qu'il eut fait la guerre à Persée et qu'il en vint à déposer sa haine, il reçut, dit-on, de grands privilèges des Argiens, parmi lesquels cet enclos sacré exceptionnel. Plus tard, Dionysos fut appelé Krésios, parce qu'il y ensevelit Ariane, après sa mort. Lykéas raconte que lors d'une réfection du temple, on y découvrit une urne funéraire en terre cuite. C'était celle d'Ariane. Il affirme avoir vu l'urne en personne, ainsi que d'autres Argiens. Près de celui de Dionysos, il y a un temple d'Aphrodite Ourania.

Pausanias signale, d'après les sources littéraires qu'il utilise ${ }^{1}$, l'existence d'une construction souterraine interprétée par référence à trois groupes légendaires : $\left(1^{\circ}\right)$ on en fait la prison de Danaé; $\left(2^{\circ}\right)$ la tombe de Krotôpos renvoie à la légende de Linos; $\left(3^{\circ}\right)$ l'identification de la structure comme temple de Dionysos Krésios, dont l'aition est la guerre de Persée et de Dionysos. La dernière identification était celle que suivait Lykéas, un poète local féru d'érudition, qui connaissait l'attaque de Pyrrhos $(\sim 272)$ et qui n'est cité que par le Périégète $^{2}$. Il pourrait être la source unique de tout le passage. La multiplicité des explications proposées prouve qu'on se trouve devant un cas typique d'explication de curiosités (une chambre souterraine) par référence à la tradition érudite. Pausanias permet d'affirmer $\left(1^{\circ}\right)$ que l'érudition locale hellénistique ou romaine gardait le souvenir d'un culte de Dionysos Krésios, qu'on ne savait plus où localiser; $\left(2^{\circ}\right)$ qu'on en expliquait la fondation par la légende de la lutte entre Persée et Dionysos; $\left(3^{\circ}\right)$ que Lykéas expliquait l'épithète par référence à une tradition situant le tombeau d'Ariane (qu'il a même cru identifier) à Argos. Les autres monuments mis en rapport avec la légende de Persée et de Dionysos figurent parmi les curiosités que Pausanias décrit dans l'itinéraire qui conduit de l'agora à la porte d'Ilithye. Il n'y a donc pas de raison de supposer que la légende argienne différait sur ce point de celle de Nonnos, qui fait venir Dionysos du Nord. Il n'y en a pas non plus de penser qu'on avait recours à d'autres légendes pour expliquer la mort d'Ariane (katasterismos après sa mort des mains de Persée). L'identification que soutient Lykéas a pu être favorisée par l'existence, à proximité de la structure controversée, d'un temple dédié à Aphrodite Ourania, dont les liens avec Ariane sont bien connus. L'existence d'un culte d'Ariane à Argos demeure donc très sujette à caution. À l'époque de Lykéas, l'épithète, isolée, de Krésios, quel que soit son sens premier et son

1 Il se pourrait bien que la structure désignée par Lykéas comme un temple soit l'une des tombes de la Deiras (cf. J. DESHAYES, Les fouilles de la Deiras, Paris, 1966), comme le suspectaient les voyageurs du siècle dernier. Mais on ne peut le déduire du texte de Pausanlas lui-même.

2 F. JACOBY, Fr,Gr.H., 312; H. LLOYD-JONES-P. PARSONS, Supplementum Hellenisticum, BerlinNew York, 1983, p. 257-258, n 527-530. 
rapport au culte de Dionysos, n'était plus comprise que dans le cadre de la légende de la lutte entre Persée et Dionysos.

IV. Lerne (p. 223-325) était, jusqu'à la fin de l'Antiquité, un des hauts-lieux du culte de Dionysos. Dans ce site, où les cultes de la fécondité se sont succédé sans interruption depuis l'époque néolithique, des cérémonies en l'honneur de Dionysos sont attestées par Pausanias et Plutarque. Dionysos, mort ou prisonnier des enfers, est évoqué au son des trompes. La tradition argienne, dont l'iconographie prouve l'ancienneté, faisait mourir Dionysos entre les mains de Persée et mettait son tombeau à Delphes. D'autre part, les rapports entre Dionysos et Artémis expliquent l'usage qui consistait à faire venir le feu purificateur des Lernaia du sanctuaire d'Artémis Pyronia à Pheneos. La kathodos du dieu, dont les Grenouilles donnent une transposition, fait intervenir à Lerne un autre ensemble de mythes impliquant Sémélé et des traditions locales (Prosymnos/Polymnos) qui permettent de se faire une idée des rites d'initiation qu'on y accomplissait et qui pourraient avoir donné lieu à de véritables pratiques homosexuelles. Les épigrammes grecques et latines du Bas-Empire semblent indiquer l'antériorité de Dionysos sur Déméter, qui pourrait avoir pris la place d'Artémis.

Je reviens plus en détail sur les cultes de Lerne dans deux études sous presse : La mort de Dionysos à Argos (à paraître dans les actes du colloque The Role of Religion in Early Greek Polls [Athènes, Institut suédois, octobre 1992] et Le tombeau de Dionysos à Delpbes (à paraître dans les Mélanges O. Scholler, Luxembourg). Voir déjà La mort de Dionysos à Argos et son tombeau à Delphes [en polonais], in I. LEWANDOWSKI et A. WÓJCIK (éds), Vetustatis amore et studio (Mélanges C. Liman, Poznan 1995), p. 131-145. Je me contenterai ici de souligner quelques points de désaccord avec une reconstruction qui, je le répète, se signale par la richesse de son érudition.

1. À mon avis, Pausanias distingue soigneusement entre l'initiation qu'il

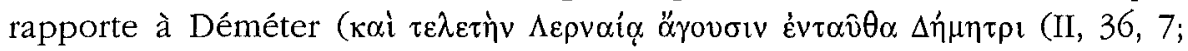

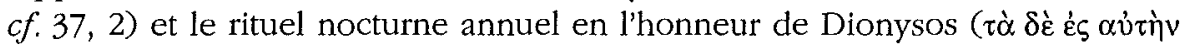

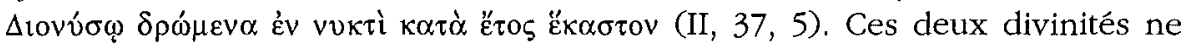
sont d'ailleurs pas les seules à se partager le site sacré de Lerne: Athéna et Aphrodite y étaient aussi honorées.

2. La question de l'existence de rites homosexuels liés au mythe de Prosymnos/Polymnos demeure ouverte. Les rites que Pausanias a jugé impie de dévoiler sont, en partie au moins, ceux-là mêmes qu'évoquait Plutarque. Ce sont eux qui ont dû survivie jusqu'à la fin de l'Antiquité.

3. Les Argiens ont su intégrer dans leurs cycles légendaires et tourner à leur profit les récits de l'arrivée de Dionysos. Au contraire, du moins dans les limites où nous pouvons appréhender les faits, les mythes de Dionysos à Lerne, dont le caractère épichorique est assuré, apparaissent comme très isolés au milieu de légendes fortement structurées autour des pôles formés par les cycles d'Inachos et de Persée, dans le Nord de la Plaine, et de Danaos, dans la région littorale.

4. Le traitement de la légende de Déméter présente des analogies avec celui de la légende de Dionysos. À Argos, les récits de l'accueil s'insèrent parfaitement dans le cycle d'Inachos, alors que la déesse n'a aucun rapport apparent 
avec le cycle de Danaos à Lerne. Les mythes de Déméter n'y ont rien à voir non plus avec ceux de Dionysos. Les cultes de Déméter et de Dionysos dans le monde gréco-romain avaient des aspects communs. On ne s'étonnera donc pas de les voir se côtoyer dans les calendriers de culte ou dans des sanctuaires. À l'historien de préciser chaque fois par quels traits ils se rapprochent. À Lerne, ni la nature des rites, ni les récits qui les expliquaient ne corroborent la thèse d'une parenté structurelle entre Dionysos et Déméter.

5. L'institution des Lernaia ( $\tau \dot{\alpha} \Lambda \hat{\varepsilon} p v \alpha 1 \alpha$ ), pour lesquels les Argiens se procuraient le feu nécessaire dans le sanctuaire d'Artémis Pyronia (Paus., VIII, 15, 9) concernent la seule Déméter. Le rite ne peut donc s'expliquer par des relations privilégiées d'Artémis et de Dionysos. Artémis paraît d'ailleurs absente de Lerne; son rôle de détentrice du feu purificateur suffit à lui seul à rendre compte du rite.

6. Le seul mythe que, dans l'état actuel de nos connaissances, nous puissions rapporter au tombeau de Dionysos à Delphes est celui de son démembrement par les Titans. La légende reliant la mort de Dionysos, fils de Sémélé, des mains de Persée et son ensevelissement à Delphes a été élaborée dans le cadre d'un récit suivi de ses divers exploits. Elle ne saurait être antérieure à l'époque hellénistique. Dans la forme sous laquelle elle nous est parvenue, elle n'a d'ailleurs pas été rédigée avant le premier siècle après J.-C. Il se peut qu'elle porte la marque de Céphalion, dont on sait par ailleurs qu'il a donné une version rationalisante de la geste de Dionysos. Toute les allusions qu'y font les auteurs chrétiens dépendent d'une notice rédigée par Sextus Julius Africanus pour sa chronique et dont Eusèbe de Césarée avait donné une version abrégée. Cette notice évbémériste attribuait à un poète inconnu de nous, Deinarchos, le récit de la mise à mort de Dionysos par Persée. Elle mentionnait peut-être Céphalion à propos du tombeau de Delphes et suivait Philochore, mais sur un seul point : pour expliquer le fait que Dionysos entrainait des femmes dans son armée. 